\title{
RF CMOS Wireless Implantable Microsystem for Sacral Roots Stimulation with On-Chip Antenna and Far-Field Wireless Powering
}

\author{
F. Rodrigues, S. Gomes, P. Anacleto, J. Fernandes, P. M. Mendes \\ Dept. of Industrial Electronics, \\ University of Minho, \\ Guimarães, Portugal
}

\begin{abstract}
The use of heterogeneous integration technologies is the path for the development of further miniaturized, smarter, and energy autonomous microdevices, which are required to tackle the challenge of monitoring and/or control the health condition of everyone, everywhere. The interaction with human body requires the use of flexible materials, while the electronic component are based on rigid materials, like silicon substrates. Also, once inside the human body, it is desirable to have a wireless link for data communication, as well for RF powering, using energy harvesting techniques or dedicated powering RF links. This paper shows the design of an implantable microsystem to be used for functional electrical stimulation of sacral roots. The proposed system includes flexible electrodes, integrated with an RF CMOS chip, which is powered by a wireless link through an efficient on-chip antenna.
\end{abstract}

Keywords-RF CMOS; wireless power; energy harvesting; biomedical device; implantable microsystem;

\section{INTRODUCTION}

The healthcare sector is under high pressure to release new solutions, procedures and devices that allow better and cheaper treatment and diagnostic. There is a trend to use alternative healthcare solutions to pills, like electrical stimulus of neural system, or the brain initiative pushing for smaller and smarter microsystems with increased autonomy. The main goal is to have everything integrated, where a chip, preferably CMOS, due to well-known reasons of integrability and cost, is the core of all system.

Lower urinary tract disorders (e.g., incontinence) are among the most traumatic consequences of spinal cord injuries. Functional electrical stimulation (FES) can be used to restore normal urinary functions in spinal cord injured people. Sacral roots have been widely reported as a good site to elicit bladder voiding via electrical stimulation [1] [2].

However, electrical stimulation of sacral nerve roots first excites larger fibers innervating urethral sphincter and then parasympathetic innervating detrusor muscle. This recruitment order often results in simultaneous activation of sphincter and detrusor leading to muscle fatigue and bladder/sphincter dyssynergia.

Multipolar cuff electrodes can be used to enhance selective

This work was supported by the Portuguese Foundation for Science and Technology (SFRH/BD/62608/2009), FCT-PTDC/EEI-TEL/2881/2012, Programa Operacional Temático Fatores de Competitividade (COMPETE) and Fundo Comunitário Europeu FEDER. stimulation in peripheral nerves [3]. In spite of their widespread use in other peripheral nerves, application of multipolar electrodes for selective stimulation in small S2-S4 sacral nerve roots is still lacking. Indeed, designing multipolar cuff electrodes for selective stimulation of sacral nerve roots is a challenge because of their diameter range, which can be as low as $500 \mu \mathrm{m}$ [4]. Miniaturization through integration is a key issue to obtain smarter systems required to meet the demands on several areas, mainly in the biomedical field.

In this way, new solutions are required to be used in the medical sensing and actuating field, namely ultraminiaturized solutions able to interact with the nervous system. In this paper, we show a solution to fabricate a wirelessly powered microsystem able to interface with the neural network, where the system is fully built on a silicon wafer. Fig. 1 shows the fabricated microsystem.

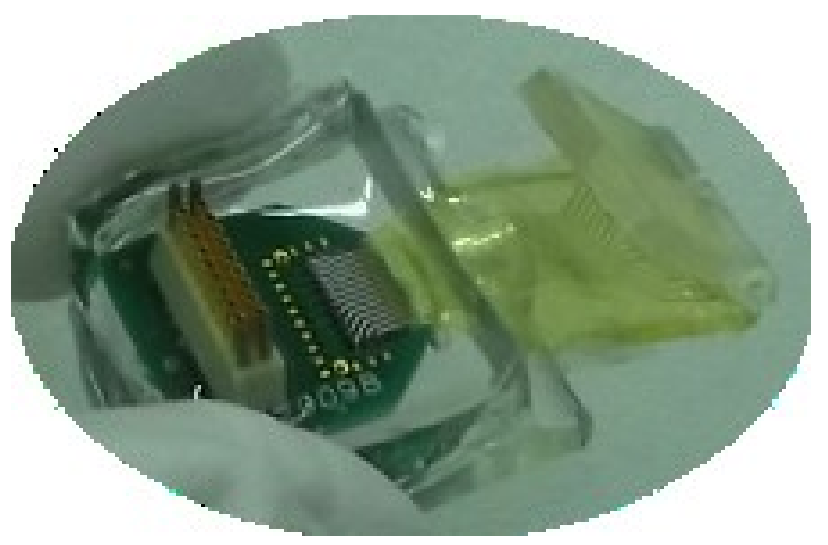

Fig. 1. Fabricated microdevice for sacral roots stimulation.

\section{SYSTEM INTEGRATION}

The fig. 2 shows the three main components of the proposed system and the overall dimensions. The main drawback for this system is that it would require the use of wires between the implanted device and the outside world. This paper proposes a solution to avoid PCB and wirebonding. 


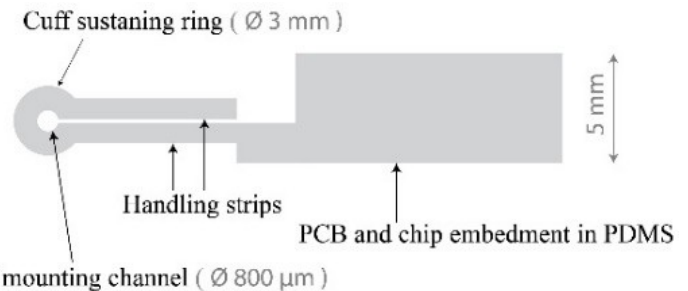

Cuff mounting channel ( $\varnothing 800 \mu \mathrm{m}$ )
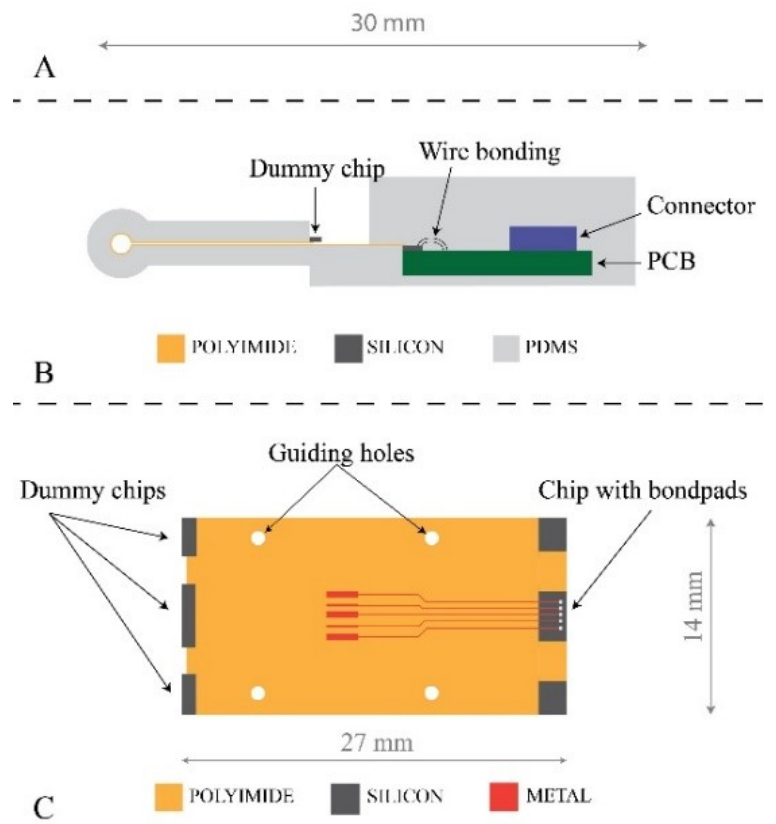

Fig. 2. Artistic view for a highly integrated microsystem used for sacral nerve stimulation.

\section{A. Neural interface overall integration}

Fig. 2 a) and b) shows the neural interface overall integration methodology, as well as the main elements. The first element is the cuff electrode, which is made on a flexible material. The second is a silicon chip, which is integrated with the flexible material and is required to make the interface between the microelectrodes and stimulation device. The third element is the PCB plus connector, which is the way to the stimulator. Finally, all the system must be packaged using a biocompatible material. In our case, the final fabrication step is packaging with PDMS. The PDMS package is used with two purposes: to isolate the microdevice from the body and to be used as a handling mechanism during the surgery when the device is implanted.

\section{B. Electrodes}

Fig. 2 c) shows the solution used to interconnect the silicon chip with the sacral roots. This is achieved using a microprocessing technique that allows the integration of a flexible material with a silicon wafer. With this technique it's possible to attend two goals: the good contact at the interface between the electrode and the neural system, since the electrode will be around the nerve; and the possibility to have, instead of the standard cuff electrode with three contacts, a multisite electrode, enabling the selective stimulation of sacral nerve roots through spatial selectivity [4].

\section{Wireless Interface}

The proposed microsystem must be powered using a dedicated RF link, or harvest the surrounding RF energy, and should offer a wireless data link. That implies the use of RF circuitry for energy and communications, as well as an antenna. Since we want a system as small as possible, the silicon chip (chip with bondpads in Fig. 2 c)) should be a RF CMOS chip (our design was made using $180 \mathrm{~nm}$ MM/RFCMOS form UMC), where all the RF and non-RF electronics will be implemented. Also, the antenna should be integrated within the overall system. Nowadays, the available solutions for wirelessly power implanted devices rely mainly on inductive links to power the in-body device, which requires that the out-body device is properly very well positioned and attached to the body [7].

However, desirably, the outside device should be able to move inside a certain volume, without affecting the link performance significantly. That requires a design based on a far-field [8] link rather then on near field (inductive or radiative). Since the far-field solutions will deliver less power, there are two main implications when using this approach: the antenna on the in-body device should be as efficient as possible (new antenna designs and integration methods are required), and the power available to operate the full device is less, which requires solutions that are able to harvest that power and that are able to operate with less power. The antenna integration, on top of a silicon wafer, is based on self-folding fabrication technology $[6,9]$. The solution proposed for antenna integration was designed to allow the integration of antennas with improved radiation efficiency, maximizing the overall RF link efficiency.

\section{WIRELESS LINK DESIGN}

After solving the integration issues and having the possibility to fabricate a highly integrated and miniaturized device, one key issue is to design the RF to DC circuitry that will convert and manage the power collected by the antenna. In this way, it is necessary to investigate how much power is possible to be received inside the chip and to design the RF CMOS circuitry that is able to operate on that power.

\section{A. Wireless link sharing}

Since we require a wireless link for power and for data, two options may be considered. Both paths may share the same antenna (actually we will have three paths: RF power, data reception and data transmission) or the system uses two different antennas. Since the proposed integration methodology allows to easily integrate the antenna, instead of using filters and/or switches we propose the use of two antennas, with separated functions. There are three main reasons to use this solution: we want to continuously power up the device, while receiving a signal, to avoid extra losses due to switch, and to avoid power consumption from the switch.

\section{B. Wireless energy receiver}

The RF to DC converter implemented was adapted from [8]. Fig. 3 shows the implemented circuitry on chip. The decision to use three stages was made based on the expected energy that will be available on-chip, supplied by the RF powering link. 


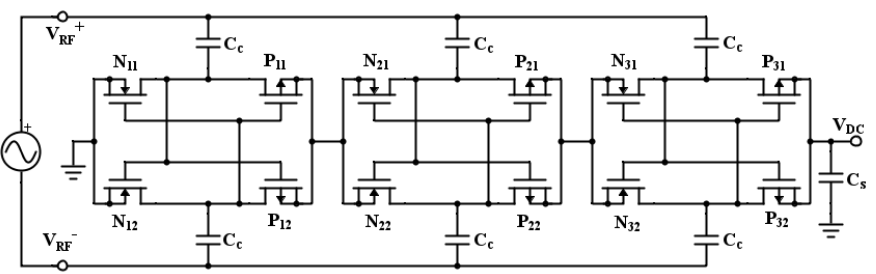

Fig. 3. RF to DC conveter, implemented on $180 \mathrm{~nm} \mathrm{MM/RFCMOS} \mathrm{UMC.}$

After the design of the RF to DC converter, a current control device was required, either to charge a small battery or a supercapacitor, or to control the current supplied to the electronic circuit responsible for the pulses that are generated to trigger the sacral roots. Fig. 4 shows the implemented circuit based in the work present at [9].

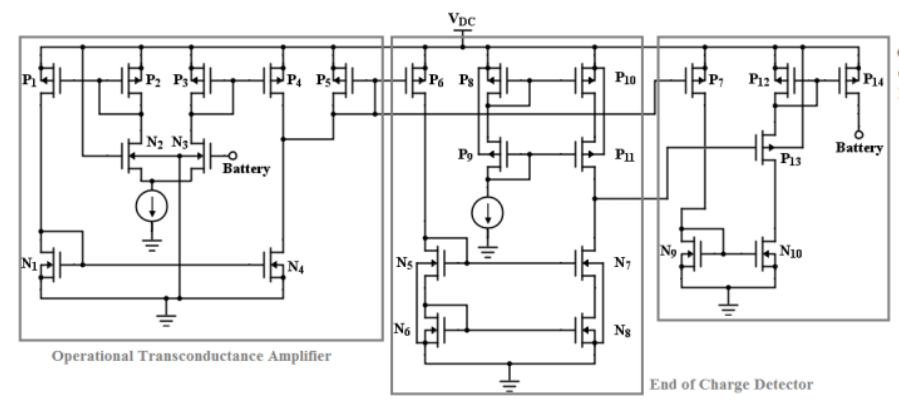

Fig. 4. CMOS battery charger, implemented on $180 \mathrm{~nm}$ MM/RFCMOS UMC.

\section{Wireless transmitter}

For this system, since the data that is sent from the chip is minimal, a simple VCO was designed that uses a ring oscillator with current control for frequency tuning.

\section{RESUlTS AND DISCUSSION}

After all the previous considerations a chip layout was designed, as shown in fig. 5 .

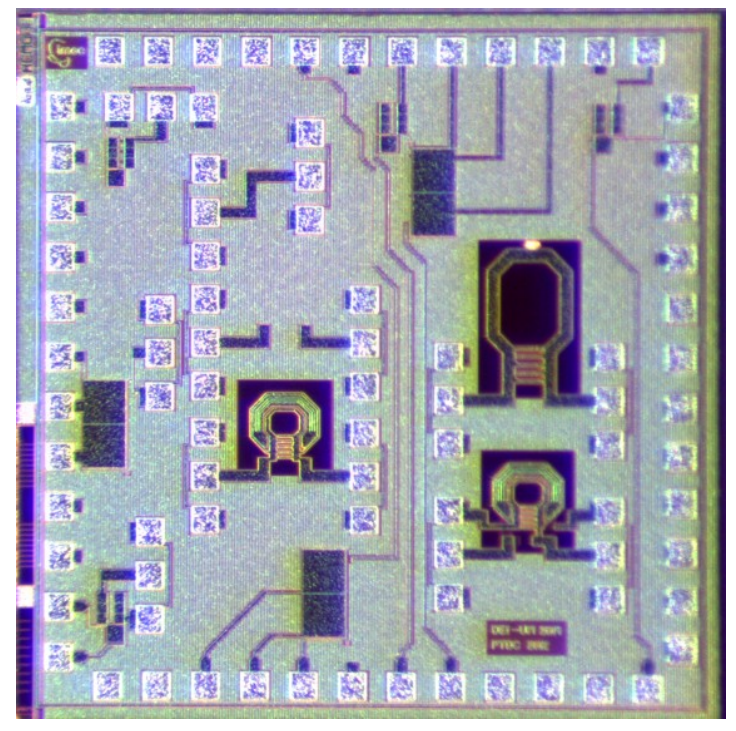

Fig. 5. Fabricated $180 \mathrm{~nm} \mathrm{MM/RFCMOS} \mathrm{microphotograph.}$
Since it is still a prototype, besides the RF to DC converter, the power control, the VCO, many RF test stuctrures and some control electronics where included for testing purposes. To test the effectiveness of all the integration methodology it was required to test two different aspects: one was to verify the ability to transmit power enough to the implanted device, using the proposed integration methodology for antennas; and the other was to verify how much power would be required to operate the RF CMOS circuitry that would be responsible for $\mathrm{RF}$ to DC conversion, as well the controlling circuitry for battery charging.

\section{A. Power reception}

In order to characterize the potential of the proposed system to establish a wireless link for powering the RF CMOS circuitry, a transmitting antenna, with a gain of $10 \mathrm{~dB}$, was placed $30 \mathrm{~cm}$ apart of the antenna of system, so that the RF energy transmission would take place in the far-field. With that setup, it was possible to transmit enough power to light up a LED. The graph shown in Fig. 6 illustrates the results of wireless power transfer to a resistive load. The rectifier's highest output voltage was $1.5 \mathrm{~V}$, at $2.3 \mathrm{GHz}$.

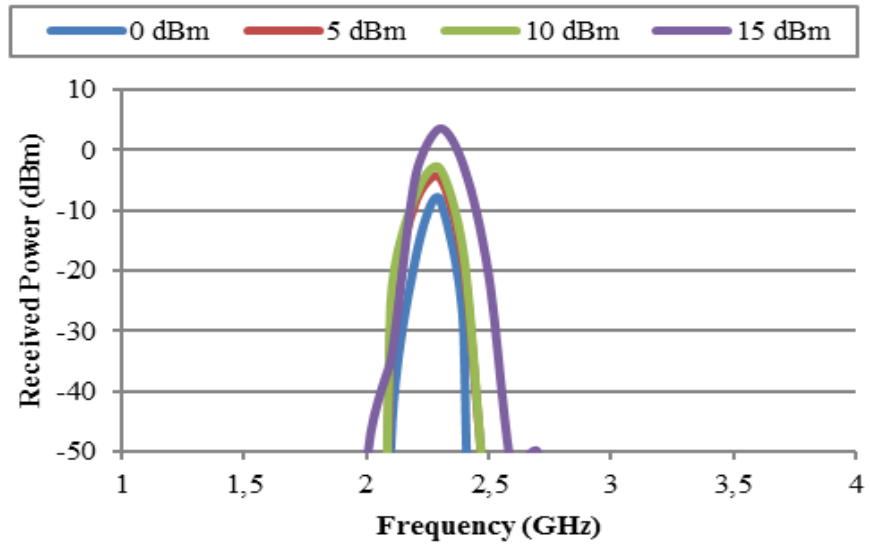

Fig. 6. Power received by the $1 \mathrm{k} \Omega$ load, from $1 \mathrm{GHz}$ to $4 \mathrm{GHz}$, for various input powers (before an amplifier with $32 \mathrm{~dB}$ of gain).

The measured wireless link efficiency (ratio between the power at the output of the transmitter antenna and power at the input of the rectifier stage) was $-36 \mathrm{~dB}$. This efficiency changes with distance (since it includes free space loss). This demonstrates that the proposed solution was effective to use the far-field approach to transfer energy to the microsystem containing a microantenna.

\section{B. On-chip RF CMOS circuitry}

After the results of Fig. 6 we know that it will be possible to have voltages above $1 \mathrm{~V}$ at the integrated antenna terminals. That will be enough to allow the operation of the on-chip RF to DC circuits. But if we don't want to send the huge amount of power we used to obtain Fig. 6, we should check how the proposed RF to DC circuitry behaves for low power levels. Fig. 7 and Fig. 8 show the voltage output of the on-chip RF to DC circuit (fig. 3 ) rectifier. 


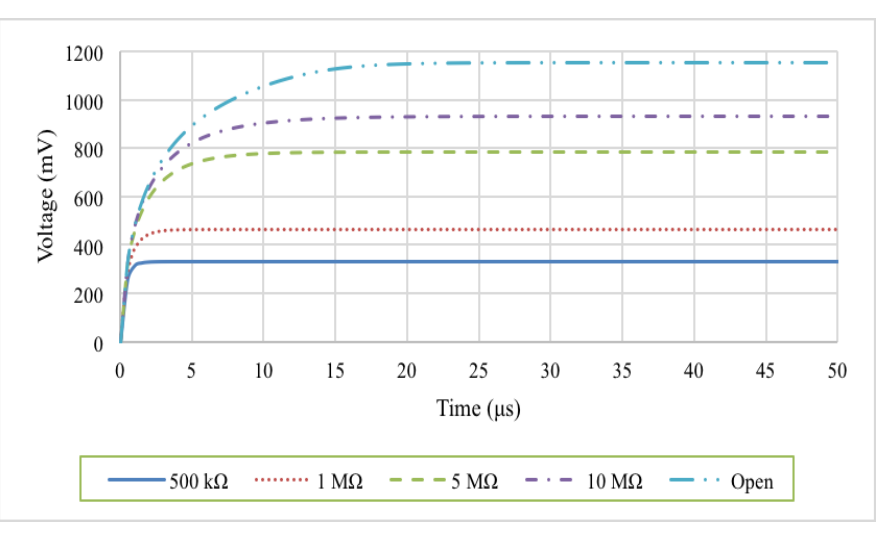

Fig. 7. DC voltage output of the RF to DC circuit for an RF input voltage amplitude of $500 \mathrm{mV}$.

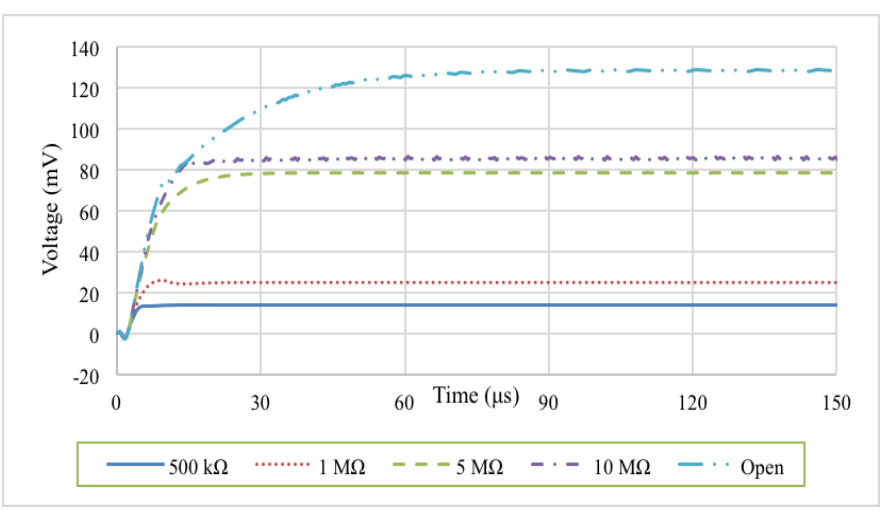

Fig. 8. DC voltage output of the RF to DC circuit for an RF input voltage amplitude of $350 \mathrm{mV}$.

These plots shows that such circuitry should see around $500 \mathrm{mV}$ at the antenna terminal for proper operation. For voltages bellow $500 \mathrm{mV}$, new circuitry must be designed.

\section{CONCLUSION}

In this paper a methodology was proposed to obtain a wireless implantable microsystem for sacral roots stimulation. It was shown that is possible to integrate a flexible electrode, with multiple contacts for selective stimulation, with a RF CMOS chip, and an efficient antenna to provide a far-field RF link for powering and data communications. An antenna was fabricated and tested, with a RF to DC circuit, to investigate if is possible to deliver enough power to the implanted device. Finally, an RF to DC and battery charger where designed on $180 \mathrm{~nm}$ CMOS.

\section{REFERENCES}

[1] Brindley, GS, An implant to empty the bladder or close the urethra. J Neurol Neurosurg Psychiatry. 1977, Vol. 40, pp. 358-369.

[2] Rijkhoff NJ, et al., Selective detrusor activation by electrical stimulation of the human sacral nerve roots. Artif. Organs. 3, 1997, Vol. 21, pp. 223226.

[3] Nielsen TN, et al., Transverse Versus Longitudinal Tripolar Configuration for Selective Stimulation With Multipolar Cuff Electrodes. IEEE Trans. on Biomed. Engineering. 2011, Vol. 58, pp. 913-919.

[4] F. Rodrigues; B. Mimoun; M. Bartek; P.M. Mendes; R. Dekker, "Flexible multipolar cuff microelectrode for FES of sacral nerve roots," $n$ Proc. 17th Annual International FES Society Conference (IFESS 2012), Banff, Alberta, Canada, pp. 1-4, Sept. 2012.

[5] F. Rodrigues, L. Goncalves, M. Bartek, B. Mimoun, A. Fouchard, P. Pham, O. David, P. M. Mendes, "Design, fabrication and modelling of a cuff electrode for peripheral nerve stimulation," In 3rd Portuguese Bioengineering Meeting, Braga, Portugal, 20-22 February 2013.

[6] D. Gracias, E. Gultepe, P. Anacleto, J. H. Correia, and P. M. Mendes, "Antenna operating frequency selection for energy harvesting on nano biomedical devices," Proc. 41 st Eur. Microw. Conf., pp. 64-66, 2011.

[7] - J. S. Ho, A. J. Yeh, E. Neofytou, S. Kim, Y. Tanabe, B. Patlolla, R. E. Beygui, and A. S. Y. Poon, "Wireless power transfer to deep-tissue microimplants," Proceedings of the National Academy of Sciences, vol. 111, no. 22, pp. 7974-7979, 2014.

[8] S. Gomes, J. Fernandes, P. Anacleto, E. Gultepe, D. Gracias, P. M. Mendes, "Ultra-Small Energy Harvesting Microsystem for Biomedical Applications," in 44st European Microwave Conference, 05-10 October, Rome, Italy, 2014.

[9] H. Dinis, P. Anacleto, J. Fernandes, P. M. Mendes, Characterization of Chip-Size Electrically-Small Antennas for Smart Wireless Biomedical Devices," The 9th European Conference on Antennas and Propagation, Lisbon, Portugal, on 12-17 APRIL 2015, Accepted

[10] K. Kotani, A. Sasaki, and T. Ito, "High-Efficiency Differential-Drive CMOS Rectifier for UHF RFIDs," IEEE Journal of Solid-State Circuits, vol. 44, no. 11, pp. 3011-3018, Nov. 2009.

[11] B. Do Valle, C. T. Wentz, and R. Sarpeshkar, "An area and powerefficient analog li-ion battery charger circuit.," IEEE Transactions on Biomedical Circuits and Systems, vol. 5, no. 2, pp. 131-137, Apr. 2011. 